\title{
The Effect of Azalea on Oxidative Stress, Immune Factors and Vasodilator Factor in Hypertensive Rats
}

\author{
Z. LIU, JING GUO', ZHENHUI DU AND ERQING CHA* \\ Cerebrovascular Disease Center, Gansu Provincial Hospital, Lanzhou, ${ }^{1}$ Radiological Department, The Second People's Hospital \\ of Jiuquan City, Gansu 735000, P.R. China
}

\section{Liu et al.: Effect of Azalea on Oxidative Stress, Immune and Vasodilator Factors}

To analyze the effects of azalea on oxidative stress, immune factors and vasodilator factors in hypertensive rats. 24 male Specific-pathogen-free grade spontaneous hypertension rats were divided into model group (normal saline), azalea group (50 mg/kg/d azalea), verapamil group (50 mg/kg/d verapamil) and 8 normal control group (normal saline) and observed the changes of tail artery systolic pressure of rats for 2-18 w after administration, superoxide dismutase, malondialdehyde were detected after 18 whe levels of nuclear factor kappa B, Smooth muscle alpha-actin and Smooth muscle alpha actin were detected by reverse transcription-polymerase chain reaction and Western blot. The systolic pressure of the caudal artery in azalea group and verapamil group was significantly lower than that in the model group (p<0.05). The levels of Malondialdehyde and reactive oxygen species in azalea group and verapamil group were significantly lower than that in the model group and Superoxide dismutase level was significantly higher than that in the model group $(p<0.05)$. The levels of interleukin-6 and tumor necrosis factor-alpha in azalea group and verapamil group were significantly lower than those in the model group $(\mathbf{p}<0.05)$. The levels of Angiotensin $\alpha$ and endothelin-1 in azalea group and verapamil group were significantly lower than that in the model group and no and nitric oxide synthase levels were significantly higher than those in the model group $(p<0.05)$. The expression of nuclear factor kappa light chain enhancer of activated $B$ cells messenger Ribonucleic acid and protein in azalea group and verapamil group was significantly lower than that in model group and Smooth muscle alpha-actin messenger Ribonucleic acid and protein were significantly higher than that in model group $(p<0.05)$. Azalea can significantly reduce the systolic pressure of hypertensive rats and protect the aortic injury. It may be related to the reduction of oxidative stress, regulation of immune factors and diastolic vasodilator factors, and regulation of nuclear factor kappa light chain enhancer of activated B cells, Smooth muscle alpha-actin expressions.

Key words: Azalea, hypertension, oxidative stress, immune factor, diastolic vasodilator

Hypertension is a high-risk factor for cardiovascular diseases such as stroke and coronary heart disease among middle-aged and elderly people. It can be accompanied by the function or organic damage of the heart, brain, kidney and other organs. If the patient does not receive timely and effective treatment, it can induce complications such as heart failure, diabetes, kidney disease and increase the risk of death of the patient. It has become a common and frequentlyoccurring disease that seriously endangers the health of the Chinese people ${ }^{[1]}$. At present, the pathogenesis of hypertension is unclear and the increased vascular oxidative stress ability has been confirmed in a variety of animal models of hypertension. It can cause changes in vascular structure and increase in peripheral vascular resistance, promote intravascular thrombosis,

*Address for correspondence E-mail: biti4036@126.com

May-June 2021 vasoconstriction and endothelial inflammation and induce cardiovascular diseases such as hypertension and diabetes ${ }^{[2,3]}$. Although the traditionally used antihypertensive drugs are effective, long-term use can cause many side effects such as liver metabolism disorders and changes in blood biochemical indicators. Therefore, exploring a safe and effective drug to prevent and treat hypertension is of great significance for reducing the incidence of cardiovascular disease.

\footnotetext{
This is an open access article distributed under the terms of the Creative Commons Attribution-NonCommercial-ShareAlike 3.0 License, which allows others to remix, tweak, and build upon the work non-commercially, as long as the author is credited and the new creations are licensed under the identical terms
} 
Rhododendron is a compound of dihydroflavonoids, which has anti-infective, antibacterial, antiviral and immunosuppressive effects. It is often used clinically to treat chronic bronchitis. It can significantly relax bronchial smooth muscles. It is speculated that it has the effect of relaxing blood vessels ${ }^{[4,5]}$. However, there are few clinical reports on the treatment of hypertension with rhododendron. Therefore, this study established a hyperbaric oxygen rat model to analyze the effects of oxidative stress levels, immune factors and vasodilatory and contractile factors in rats.

\section{MATERIALS AND METHODS}

\section{Experimental reagents and instruments:}

Rhododendron (Shanghai Chunyou Biotechnology Co., Ltd.); Verapamil (Shanghai Baoman Biotechnology Co., Ltd.); Ribonucleic acid (RNA) Extraction and Purification Kit (Nanjing Laifusai Biotechnology Co., Ltd.); Complementary DNA (cDNA) synthesis kit (Shanghai Yanhui Biotechnology Co., Ltd.); Protein extraction kit (Shenyang Wancai Biotechnology Co., Ltd.); sodium dodecyl sulfate polyacrylamide gel electrophoresis (SDS-PAGE) gel preparation kit (Beijing Soleibao Technology Co., Ltd.); nuclear factor kappa light chain enhancer of activated B cells (NFkappa B), Smooth muscle alpha-actin (SM $\alpha$ - actin) antibody (Biotech (Shanghai) Co., Ltd.).

Non-invasive blood pressure measurement system (Beijing Jiandel Technology Co., Ltd.); low-temperature high-speed centrifuge (Beijing Anmag Trading Co., Ltd.); Electric heating constant temperature water bath (Hangzhou Nordin Scientific Equipment Co., Ltd.); Microplate reader (Shanghai Fuze Trading Co., Ltd.); Micropipette (Shanghai Jingxin Industrial Development Co., Ltd.); Automatic biochemical analyzer (Nanjing Beiden Medical Co., Ltd.); Spectrophotometer (Beijing Jinda Sunshine Technology Co., Ltd.); Gel imaging system (Shanghai Jinpeng Analytical Instrument Co., Ltd.).

\section{Experimental animals and grouping:}

Twenty-four male SPF-level spontaneously hypertensive rats and 8 normal rats were selected, purchased from Beijing Weitong Lihua Laboratory Animal Technology Co., Ltd., and raised in the Animal Research Center of our hospital. The temperature is $22 \sim 24^{\circ}$, the humidity is $50 \% \sim 60 \%$, the day and night are alternated for $12 \mathrm{~h}$ and they are free to drink and ingest water. After $1 \mathrm{w}$ of adaptive feeding, they will be used for follow-up experiments. The spontaneously hypertensive rats were divided into model group, rhododendron group, verapamil group, 8 rats in each group. The normal rats were set as the normal control group to be given an equal volume of normal saline by gavage, and the model group was given an equal volume of normal saline gavage. The rhododendron group was given $50 \mathrm{mg} / \mathrm{kg} / \mathrm{d}$ rhododendron by gavage and the verapamil group was given $50 \mathrm{mg} / \mathrm{kg} / \mathrm{d}$ verapamil by gavage. The rats were administered for 2 to $18 \mathrm{w}$ to observe the changes in the systolic pressure of the tail artery. After the experiment, the rats were anesthetized by intraperitoneal injection of sodium pentobarbital, and the aortic blood was collected and the aorta was separated and stored at $-80^{\circ}$ for later use.

\section{Detection method:}

The barbital sulfate method was used to detect the superoxide dismutase (SOD) level in the aortic serum of rats. The xanthine oxidase method was used to detect the serum malondialdehyde (MDA) level, and the chemical colorimetric method was used to detect the reactive oxygen (ROS) level.

The levels of interleukin-6 (IL-6) and TNF- $\alpha$ in rat aortic serum were determined by enzyme-linked immunosorbent assay. The radioimmunoassay was used to detect the levels of Angiotensin II (Ang II) and Endothelin 1 (ET-1) in the rat aortic serum. Nitric oxide (NO) and nitric oxide synthase (NOS) activity changes were detected by nitric acid reduction method.

Detection of NF- $\kappa \mathrm{B}$ and SM- $\alpha$-actin gene expression by reverse transcription-polymerase chain reaction (RT-PCR) method: Trizol method was used to extract total RNA from rat aortic tissues and determine the content and integrity. The diluted RNA sample is reverse transcribed, and the messenger RNA (mRNA) is quantitatively analyzed by RT-PCR method. After the reaction is completed, the prepared cDNA is subjected to Polymerase chain reaction (PCR) amplification. Reaction conditions: $95^{\circ}$ and pre-denaturation for $10 \mathrm{~min}, 95^{\circ}$ for $15 \mathrm{~s}, 60^{\circ}$ for $45 \mathrm{~s}, 72^{\circ}$ extension for $2 \mathrm{~min}, 40$ cycles, $4^{\circ}$ storage, relative quantification of each gene data (data analysis with $2-\Delta \Delta \mathrm{CT}$ formula).

Detection of NF- $\kappa B$ and SM- $\alpha$-actin protein expression by Western blot method: Use Radio-Immune Precipitation Assay (RIPA) lysate to fully lyse the aortic tissue, and use Bicinchoninic acid assay (BCA) method for cell protein quantification. The protein sample was heated and denatured and separated by SDS-PAGE. After $1 \mathrm{~h}$, it was transferred to a polyvinylidene difluoride (PVDF) membrane and sealed with $5 \%$ skimmed milk 
powder. After adding the primary antibodies NF- $\kappa \mathrm{B}$ and SM- $\alpha$-actin to incubate, incubate overnight in a refrigerator at $4^{\circ}$, add the corresponding secondary antibodies and incubate at room temperature for 1 to $2 \mathrm{~h}$ and develop color by ECL chemiluminescence.

\section{Statistical methods:}

All data conforming to the normal distribution of the measurement data are represented by $(\overline{\mathrm{x}} \pm \mathrm{s})$, and the comparison between multiple groups is by single-factor analysis of variance. Student-Newman-Keuls (SNK-q) test was used for comparison between the two groups, $\mathrm{p}<0.05$ was considered as statistically different and SPSS21.0 software package was used for analysis.

\section{RESULTS AND DISCUSSION}

The systolic blood pressure of the tail artery in the model group was significantly higher than that in the normal control group at each time point $(\mathrm{p}<0.05)$. The systolic blood pressure of the tail artery of rats in the rhododendronin group and verapamil group was significantly lower than that in the model group for 6-18 $\mathrm{w}$ and the difference was statistically significant $(\mathrm{p}<0.05)$ (fig. 1).

The levels of MDA and ROS in the model group were significantly higher than those in the normal control group and the levels of SOD were significantly lower than those in the normal control group $(p<0.05)$. The MDA and ROS levels of rats in the rhododendron group and verapamil group were significantly lower than the model group and the SOD level was significantly higher than that of the model group. The difference was statistically significant $(\mathrm{p}<0.05)$ (Table 1$)$.

The levels of IL-6 and TNF- $\alpha$ in the model group were significantly higher than those in the normal control group $(p<0.05)$, the levels of IL- 6 and TNF- $\alpha$ in the rhododendron group and verapamil group were significantly lower than those in the model group $(\mathrm{p}<0.05)$ (Table 2).

The levels of Ang $\alpha$ and ET-1 in the model group were significantly higher than those in the normal control group and the levels of NO and NOS were significantly lower than those in the normal control group $(\mathrm{p}<0.05)$.

The levels of Ang $\alpha$ and ET-1 in the rhododendron and verapamil group were significantly lower than those in the model group and the levels of NO and NOS were

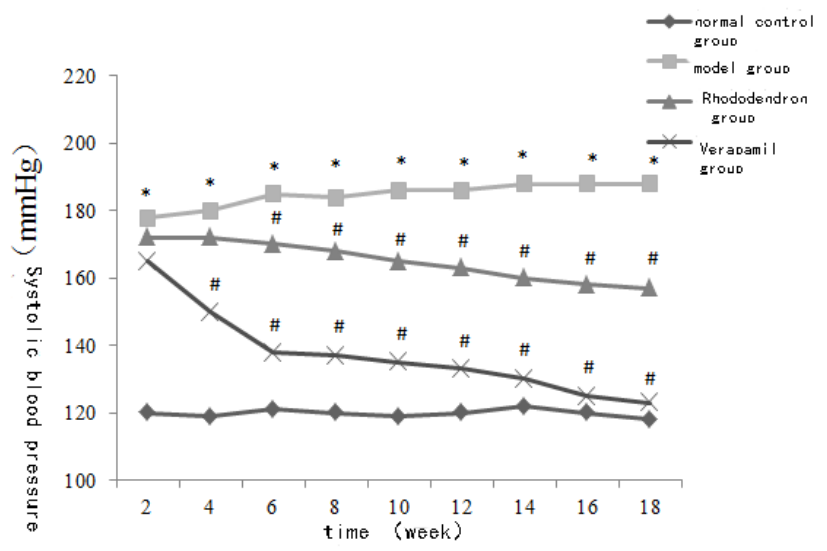

Fig. 1: The effect of rhododendron on blood pressure in hypertensive rats

Note: Compared with normal control group $* \mathrm{p}<0.05$; compared with model group $\# \mathrm{p}<0.05$

TABLE 1: EFFECTS OF RHODODENDRON ON OXIDATIVE STRESS INDICATORS IN HYPERTENSIVE RATS ( $\overline{\mathbf{X}} \pm \mathbf{s})$

\begin{tabular}{lcccc}
\hline Group & Cases & MDA $(\mathrm{nmol} / \mathrm{L})$ & SOD $(\mathrm{U} / \mathrm{mL})$ & $\mathrm{ROS}(\mathrm{U} / \mathrm{mL})$ \\
\hline Normal control group & 8 & $3.52 \pm 0.23$ & $87.75 \pm 15.19$ & $157.27 \pm 50.59$ \\
Model group & 8 & $4.72 \pm 0.29^{*}$ & $56.49 \pm 4.05^{*}$ & $566.62 \pm 84.13^{*}$ \\
Rhododendron group & 8 & $4.08 \pm 0.20^{\#}$ & $72.21 \pm 5.36^{\#}$ & $387.82 \pm 95.33^{\#}$ \\
Verapamil group & 8 & $3.50 \pm 0.31^{\#}$ & $79.36 \pm 7.10^{\#}$ & $366.24 \pm 85.74^{\#}$ \\
F & & 38.94 & 17.25 & 23.68 \\
p & & $<0.001$ & $<0.001$ & $<0.001$ \\
\hline
\end{tabular}

Note: Compared with normal control group ${ }^{*} \mathrm{p}<0.05$; compared with model group \#p $<0.05$

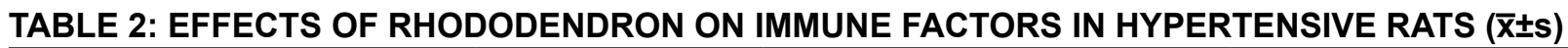

\begin{tabular}{lccc}
\hline Group & Cases & $\mathrm{IL}-6(\mathrm{pg} / \mathrm{mL})$ & TNF- $\mathrm{a}(\mathrm{pg} / \mathrm{mL})$ \\
\hline Normal control group & 8 & $79.05 \pm 4.31$ & $180.59 \pm 9.20$ \\
Model group & 8 & $117.79 \pm 6.10^{*}$ & $271.51 \pm 8.82^{*}$ \\
Rhododendron group & 8 & $98.41 \pm 4.88^{\#}$ & $238.46 \pm 11.14^{\#}$ \\
Verapamil group & 8 & $91.39 \pm 4.01^{\#}$ & $206.31 \pm 8.50^{\#}$ \\
$\mathrm{~F}$ & & 87.78 & 138.65 \\
$\mathrm{p}$ & & $<0.001$ & $<0.001$ \\
\hline
\end{tabular}

Note: Compared with normal control group ${ }^{*} \mathrm{p}<0.05$; compared with model group \#p<0.05 
significantly higher than those in the model group. The difference was statistically significant $(p<0.05)$ (Table 3).

The expression of NF- $\kappa B$ mRNA and protein in the aorta of the model group was significantly higher than that of the normal control group, and the expression of SM- $\alpha$-actin mRNA and protein was significantly lower than that of the normal control group $(p<0.05)$. The expressions of NF- $\kappa \mathrm{B}$ mRNA and protein in the rhododendron group and verapamil group were significantly lower than those in the model group, and the SM- $\alpha$-actin mRNA and protein were significantly higher than those in the model group with statistically significant differences $(p<0.05)$ (Table 4) (fig. 2).

Cardiovascular disease is one of the main causes of death and disability worldwide and it is a serious threat to human health. In view of the large side effects brought by drug treatment to people, finding effective and reasonable methods to prevent and treat cardiovascular diseases is of great significance to improve the quality of human life ${ }^{[6]}$. The isolation of drugs with activities to prevent and treat cardiovascular diseases from animals and plants has become a research hotspot at home and abroad. Rhododendron is naturally present in rhododendrons and other plants of the rhododendron family. Polyphenols are very diverse in structure and widely distributed in nature. They have anti-asthmatic, antitussive and expectorant effects on the respiratory system, and have vasodilator and diuretic effects on the cardiovascular system ${ }^{[7]}$. In recent years, studies have shown that it also has anti-inflammatory, immune regulation and vascular smooth muscle proliferation inhibitory effects ${ }^{[8]}$. Some scholars have found through animal experiments that for rats pre-contracted by potassium chloride, rhododendron can inhibit the intact endothelium and the de-endothelial vascular ring of rats in a concentration-dependent manner. Therefore, it is speculated that rhododendron can relax blood vessels by acting on the vascular endothelium ${ }^{[9]}$. In this study, spontaneously hypertensive rats were used as experimental animal models. The results showed that the systolic blood pressure of the tail artery in the rhododendron group was significantly lower than the model group $(\mathrm{p}<0.05)$. This shows that rhododendron can significantly reduce the systolic blood pressure of hypertensive rats.

Oxidative stress-induced vascular endothelial cell damage is an important factor in inducing a variety of cardiovascular diseases. Vascular endothelial cells can produce ROS under the action of risk factors to cause continuous increase in blood pressure and vascular damage. In addition, in patients with hypertension, the activity of antioxidant enzymes such as SOD and catalase is decreased, which can cause excessive generation of free radicals and increase the accumulation of lipid oxidation products such as $\mathrm{MDA}^{[10]}$. Therefore, serum MDA and SOD can reflect the degree of lipid peroxidation and the body's oxidative

\begin{tabular}{|c|c|c|c|c|c|}
\hline Group & Cases & Ang II $(\mathrm{pg} / \mathrm{mL})$ & ET-1 (pg/mL) & NO $(\mu \mathrm{mol} / \mathrm{L})$ & NOS $(\mathrm{U} / \mathrm{mL})$ \\
\hline $\begin{array}{l}\text { Normal control } \\
\text { group }\end{array}$ & 8 & $23.56 \pm 1.75$ & $19.56 \pm 2.47$ & $61.02 \pm 5.13$ & $40.16 \pm 4.45$ \\
\hline Model group & 8 & $42.57 \pm 2.05^{*}$ & $44.15 \pm 2.80^{*}$ & $38.19 \pm 3.88^{*}$ & $20.90 \pm 2.31^{*}$ \\
\hline Rhododendron group & 8 & $30.22 \pm 1.26^{\#}$ & $30.72 \pm 1.83^{\#}$ & $54.79 \pm 4.65^{\#}$ & $29.15 \pm 3.24^{\#}$ \\
\hline Verapamil group & 8 & $26.72 \pm 2.13^{\#}$ & $22.23 \pm 2.31^{\#}$ & $63.40 \pm 3.84^{\#}$ & $39.57 \pm 3.45^{\#}$ \\
\hline $\mathrm{F}$ & & 165.65 & 173.17 & 53.20 & 57.09 \\
\hline $\mathrm{p}$ & & $<0.001$ & $<0.001$ & $<0.001$ & $<0.001$ \\
\hline
\end{tabular}

Note: Compared with normal control group ${ }^{*} \mathrm{p}<0.05$; compared with model group \#p $<0.05$

TABLE 4: THE EFFECT OF RHODODENDRON ON THE EXPRESSION OF NF-KB AND SM-A-ACTIN MRNA AND PROTEIN IN THE AORTA OF HYPERTENSIVE RATS ( $\overline{\mathbf{x}} \pm \mathbf{s})$

\begin{tabular}{lccccc}
\hline Group & Cases & \multicolumn{2}{c}{ NF-KB } & \multicolumn{2}{c}{ SM-a-actin } \\
Normal control group & & mRNA & protein & mRNA & protein \\
Model group & 8 & $100.00 \pm 10.23$ & $0.18 \pm 0.05$ & $100.00 \pm 10.02$ & $0.59 \pm 0.12$ \\
Rhododendron group & 8 & $225.36 \pm 24.36^{*}$ & $0.56 \pm 0.07^{*}$ & $45.63 \pm 5.24^{*}$ & $0.17 \pm 0.07^{*}$ \\
Verapamil group & 8 & $148.67 \pm 12.15^{\#}$ & $0.20 \pm 0.06^{\#}$ & $68.47 \pm 12.58^{\#}$ & $0.45 \pm 0.20^{\#}$ \\
F & 8 & $122.02 \pm 14.36^{\#}$ & $0.17 \pm 0.05^{\#}$ & $70.68 \pm 10.88^{\#}$ & $0.42 \pm 0.18^{\#}$ \\
p & & 90.86 & 55.07 & 39.32 & 10.66 \\
\hline
\end{tabular}

Note: Compared with normal control group ${ }^{*} \mathrm{p}<0.05$; compared with model group \#p<0.05 

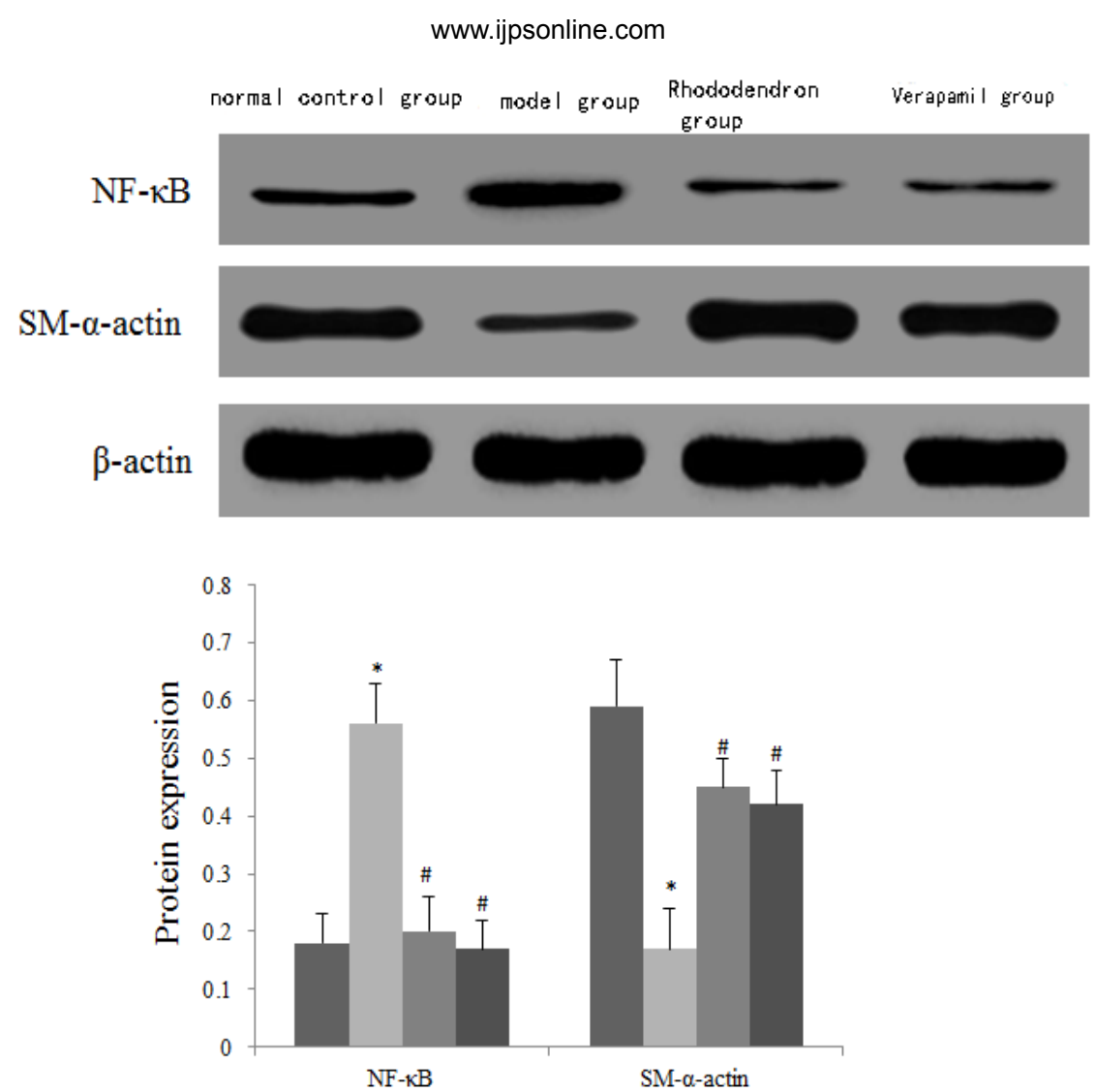

Fig. 2: Western blot detection of NF-кB and SM- $\alpha$-actin protein expression

stress state. IL- 6 and TNF- $\alpha$ are common inflammatory regulators. IL- 6 can regulate the release of cytokines and immune response in the state of hypertension; TNF- $\alpha$ can stimulate cell proliferation, differentiation and the expression of adhesion molecules, which can lead to the occurrence and development of a variety of autoimmune diseases ${ }^{[11,12]}$. ET-1 and NO are vasodilatory and contractile factors produced by vascular endothelial cells. The biological effects of the two factors are opposite. In hypertensive patients, vascular endothelial injury releases a large number of free radicals. And inhibit the synthesis and release of NO by endothelial cells, while the synthesis of Ang $\alpha$ and ET-1 are relatively increased, which further aggravates the obstacles of endothelial function ${ }^{[13]}$. The results of this study show that rhododendron can significantly reduce the levels of MDA, IL-6, TNF- $\alpha$, Ang $\alpha$, ET1 , and increase the levels of SOD, NO and NOS in hypertensive rats. It can be seen that rhododendron can protect the aorta from hypertensive rats by reducing the level of oxidative stress, regulating immune factors and diastolic and vasoconstrictor factors.

$\mathrm{NF}-\kappa \mathrm{B}$ is a gene multi-directional transcription factor widely found in eukaryotic cells. After activation, it can stimulate the expression of pro-inflammatory factors such as TNF- $\alpha$, and participate in the occurrence and development of various cardiovascular diseases such as hypertension and coronary heart disease ${ }^{[14]}$. SM- $\alpha-$ actin is a contraction regulatory protein and an early marker gene of differentiated vascular smooth muscle. Relevant data show that increased expression of SM$\alpha$-actin indicates that vascular smooth muscle cells are in a differentiated state ${ }^{[15]}$. The results of this study showed that the expression of NF- $\mathrm{BB}$ mRNA and protein in the rhododendron group and verapamil group was significantly lower than that of the model group and SM- $\alpha$-actin mRNA and protein were significantly higher than that of the model group $(\mathrm{p}<0.05)$. It can be seen that the protective effect of rhododendron against aortic injury in hypertensive rats is closely related to regulating the expression of NF- $\mathrm{KB}$ and SM- $\alpha$-actin.

In summary, rhododendron can significantly reduce the systolic blood pressure of hypertensive rats and protect the aorta from injury. It may be related to reducing the level of oxidative stress, regulating immune factors and vasodilatory and contractile factors, and regulating the expression of NF- $\kappa B$ and $S M-\alpha$-actin.

\section{Ethical approval:}

This study was reviewed and approved by the Ethics Committee for Experimental Animals of Gansu Provincial Hospital (ID: GPH1201), and all mice were 
treated according to the guidelines of principles of laboratory animal care (NIH publication No. 86-23, revised 1985).

\section{REFERENCES}

1. Portelli M, Papageorgiou PN. An update on idiopathic intracranial hypertension. Acta Neurochir 2017;159(3):491-9.

2. Muñoz Maniega S, Chappell FM, Valdés Hernández MC, Armitage PA, Makin SD, Heye AK, et al. Integrity of normalappearing white matter: influence of age, visible lesion burden and hypertension in patients with small-vessel disease. J Cereb Blood Flow Metab 2017;37(2):644-56.

3. Zhou Y, Wang Y, Wang X, Tian X, Zhang S, Yang F, et al. The Protective Effects of K-Opioid Receptor Stimulation in Hypoxic Pulmonary Hypertension Involve Inhibition of Autophagy Through the AMPK-MTOR Pathway. Cell Physiol Biochem 2017;44(5):1965-79.

4. Kun Z, Xiaojiang Q, Xiaomin H. Rhododendron inhibits the proliferation of VSMCs induced by Ang II by reducing the expression of Cx43. Chin Pharmacol Bull 2018;34:401-7.

5. Xiaomin H, Mingsheng Z, Xiaojiang Q. Rhododendron inhibits nicotine-induced pulmonary artery smooth muscle cell proliferation by enhancing the expression of Kv1.5 and Kv2.1. Chin J Pathophysiol 2019;35:1276-82.

6. Cooper R. Hypertension, Genes, and Environment: Challenges for Prevention and Risk Prediction. Circulation 2018;137 :662-4.

7. Dong L, Yang L, Liu F, Zhan H, Chen X. Farrerol ameliorates diabetic hepatopathy in rat model of type 2 diabetes mellitus via modulation of oxidativeinflammatory stress. Trop J Pharm Res 2020;19(1):71-76.
8. Dede E, Genc N, Elmastas M, Aksit H, Erenler R. Chemical Constituents Isolated from Rhododendron ungernii with Antioxidant Profile. Nat Product J 2019;9(3):238-43.

9. Qin X, Hou X, Li Q. The diastolic effect and mechanism of rhododendron on isolated rat aorta. J Integrate Chin Western Med Cardio-Cerebrovasc Dis 2012;10:457-8.

10. Arezu J, Asghar R, Jalil F, Asadollahi K, Ahmadi MRH. Curcumin in combination with triple therapy regimes ameliorates oxidative stress and histopathologic changes in chronic gastritis- associated helicobacter pylori infection. Arq Gastroenterol 2017;54:177-82.

11. Noori NM, Shahramian I, Teimouri A, Keyvani B, Mahjoubifard M. Serum Levels of Tumor Necrosis Factor- $\alpha$ and Interleukins in Children with Congenital Heart Disease. J Tehran Heart Cent 2017;12(1):15-22.

12. Schlosser K, Taha M, Deng Y, Jiang B, McIntyre LA, Mei SH, et al. Lack of elevation in plasma levels of pro-inflammatory cytokines in common rodent models of pulmonary arterial hypertension: questions of construct validity for human patients. Pulm Circ 2017;7(2):476-85.

13. Min L, Tian L, Peiying Z, Zhang Q, Lu L, Wang Z. hsCRP and ET-1 expressions in patients with no-reflow phenomenon after Percutaneous Coronary Intervention. Pak J Med Sci 2017;33(4):920-5.

14. Afonina IS, Zhong $Z$, Karin M, Beyaert R. Limiting inflammation - the negative regulation of NF- $\kappa \mathrm{B}$ and the NLRP3 inflammasome. Nat Immunol 2017;18(8):861.

15. Yu T, Zheng Y, Wang Y, Xiong W, Lin L. Advanced glycation end products interfere with gastric smooth muscle contractile marker expression via the AGE/RAGE/NF- $\mathrm{BB}$ pathway. Exp Mol Pathol 2017;102(1):7-14. 